Proceedings of the 2012 Winter Simulation Conference

C. Laroque, J. Himmelspach, R. Pasupathy, O. Rose, and A.M. Uhrmacher, eds

\title{
ADVANCEMENT SIMULATION OF TUNNEL BORING MACHINES
}

\author{
Tobias Rahm \\ Kambiz Sadri \\ Christian Koch \\ Markus Thewes \\ Markus König \\ Ruhr-Universität Bochum \\ Universitätsstraße 150 \\ D-44801 Bochum, GERMANY
}

\begin{abstract}
In mechanized tunneling a significant loss of performance resulting from weak spots in the supply chain or unforeseen geological conditions is a frequent and costly problem. Furthermore, disturbances of critical machine components can have such impact on the production that unforeseen modifications become necessary. Due to the sequential character the malfunction of one element might evoke cascading-effects which may result in a complete standstill of the tunneling progress. Transparent evaluation of applicable tunnel boring machine designs is essential in order to improve the productivity, avoid unplanned interruptions and to estimate the project duration in general. In order to meet these defiances, this paper presents a multi-method simulation model to investigate the advancement rate of tunnel boring machines. Processrelated disturbances can be considered easily within the presented simulation model. Simulation experiments demonstrate the purposive functionality of the model and visualize the significant influence of technical failure on the overall project performance.
\end{abstract}

\section{INTRODUCTION}

Tunnel projects vary significantly regarding dimensions, geotechnical formations, ground water table and other factors. In addition to that, tunneling in urban areas is highly influenced by the need to avoid impacts on existing buildings and infrastructure due to the excavation process. To achieve this goal, tunneling with a tunnel boring machine (TBM), which has an active face support, is a very common construction method (Maidl et al. 2012). The design and construction of a machine must consider numerous project specifications, geological conditions, and the diameter of the designed tunnel (Thewes 2007, Thewes 2010). The very high initial costs of tunneling projects result primarily from the combination of the described degree of individual requirements, the complexity of the TBM system, and the sometimes enormous dimensions. In the course of TBM projects, a change of project conditions, either evoked by unforeseen ground situations or by deficiencies in logistical processes, is frequently observed. In case the anticipated production rate of a tunneling system cannot be sustained, very high follow-up costs might arise. Furthermore, the sequential character of the production sequence of excavation and installation of lining entails the issue that disturbances and downtimes of single elements have significant influence on the performance of their successors. However, prolongations may result from failure of machine components, wear processes or organizational deficiencies. These disturbances can have such a big impact on the TBM production sequence, that further modifications of the tunneling system are necessary. Therefore, the overall performance of the machine is not determined by one single process, but rather to their appropriate interaction and the coordination of required supplies as provided by the supply chain. 
Additionally, the influence of disturbances must be investigated and their occurrence reduced to a minimum by a robust and sophisticated machine design.

Within this paper, an approach is presented to analyze production and supply chains under consideration of typical disturbances of TBM systems. The Earth Pressure Balance Shield machine (EPB-shield) is the most widely used TBM type with many recent innovations (Herrenknecht et al. 2011) and for this reason in the focus of this publication.

\section{MACHINE TECHNOLOGY}

When tunneling in unstable soft ground with shield machines is performed, it is of essential importance to support the tunnel face and avoid settlements at the surface. In order to do so, a face support pressure counteracts the earth and water pressure at the tunnel face. EPB-shields use the excavated spoil, which is pressurized in the excavation chamber, as a support medium. The main application area of EPB-shields is cohesive soil with good plastic properties (Maidl et al. 2012). To extend the application range into noncohesive grounds, a modification of ground properties by conditioning might be necessary to generate adequate characteristics for the use as support medium (Budach et al. 2010).

The production cycle of an EPB-shield can be described with seven key elements (see Figure 1). The thrust cylinders (4) use the last segment ring as abutment to press the machine forward into the ground by the equivalent length of one segment ring (7). The soil is excavated by the tools on the rotating cutting wheel (1), conditioned and finally enters the excavation chamber (2) through openings in said cutting wheel. The bulkhead (3) separates the pressurized excavation chamber from the atmospheric working conditions of the tunnel itself. The excavated ground is removed from the excavation chamber by a screw conveyor (5) and transported to the surface jobsite via belt conveyors. Since the machine operates in unstable ground conditions, the excavated tunnel must be secured by a ring of reinforced concrete segments (7). The prefabricated segments are transported to the EPB-shield usually by train or so-called multi service vehicle and then placed by an erector (6). After the thrust cylinders retracted, the erector takes one segment and assembles it to a complete ring. To avoid settlements at the surface and water ingress, a backfill material is pressed in the annular gap between soil and assembled ring. After one ring has been built, another cycle starts with the excavation of soil.

Taking an approach from a process simulation point of view, shielded tunneling consists of four main process chains: ground excavation, ringbuild, supply with required materials and landfill. Excavation and ringbuild are the core processes of mechanized tunneling. The supply and disposal of materials are part of the supply chain. Core processes are performed by the TBM. The progress of one core process affects the other and in addition to that the processes of the supply chain. The core process chains are sequential and cannot be operated simultaneously. One process must be finished in order to start the other. However, supply chain processes operate in parallel to core processes. According to the sequential character of the core processes, cascading effects have significant influence on the overall performance and efficiency of the EPB-shield. In particular, the disturbance of a single element may cause a standstill of the whole project, including loss of time and money, and must therefore be avoided if possible. 


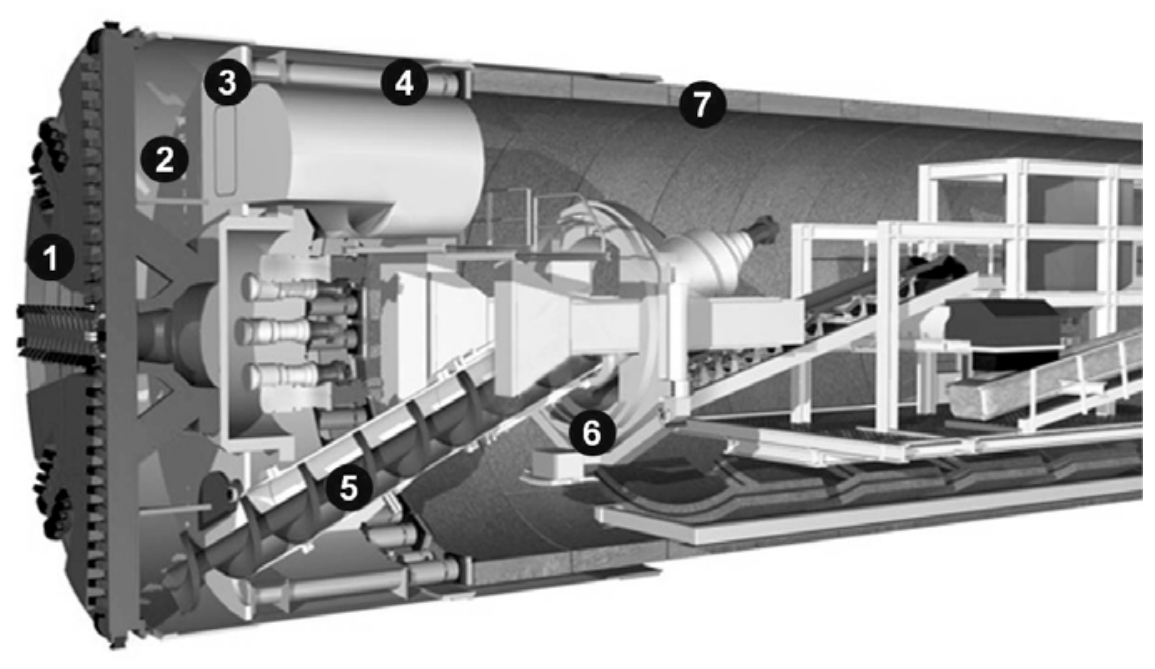

Figure 1: Earth Pressure Balance Shield machine (Herrenknecht AG 2008)

\section{SIMULATION IN MECHANIZED TUNNELING}

Most operational simulation models developed so far concentrate on the prognosis of the excavation speed in different or fuzzy soil conditions (Alvarez Grima et al. 2000, Benardos et al. 2004, Chung et al. 2006, Likhitruangsilp et al. 2003 and Ourdev et al. 2007). Apart from that, only few approaches were developed to estimate the project duration of a TBM project. Ruwanpura (2001) presented a special purpose simulation template that determines the duration of processes by distributions and links these to cost factors. Unfortunately, unplanned downtimes are not discussed in detail. Another approach was developed by Leitner et al. (2005), to easily foresee the duration of the excavation considering standstills for maintenance of the excavation tools. A limitation of this approach is that the duration of the ringbuild, as a fundamental process within the advancement cycle, can only be added as a mean value. Disturbances of this core process are not treated explicitly. Only recently, Donghai et al. (2010) presented a simulation model for mechanized tunneling in hard rock. This model also considers fundamental processes of the supply chain (e.g. muck handling). However, it is not applicable for machines operating in soft grounds and the model was very simplified due to the very high level of abstraction of the processes.

All approaches were developed to prognosticate the duration of a tunneling project, but none analyzes the processes of the machine or the supply chain in detail. Operational dependencies or cascading-effects evoked by disruption of a single machine element were not focus of research so far.

\section{TBM SIMULATION MODELING}

This section describes the development of an appropriate simulation model for mechanized tunneling in soft ground. As a first step, machine components related to the production sequence and supply chains as well as performance determining factors resulting from the interaction between machine and subsoil are classified. When dealing with a system as complex as a TBM, the need for a sophisticated, formal description method becomes obvious. The System Modeling Language (SysML) can be used to describe complex (physical) systems (Object Management Group 2010). The basic structural diagram is the socalled block definition diagram $(b d d)$ which reveals the (hierarchical) composition of a system based on its intrinsic elements. Every element of the system is represented by a distinct block. 
Figure 2 displays the $b d d$ of an EPB-shield. The first hierarchical order is established by the distinction of EPB-head (location where core processes are performed), the backup system (subterranean end of supply chain) and sub-surface construction site (aboveground end of supply chain). Additionally, the EPB-head is broken down into its assembly groups.

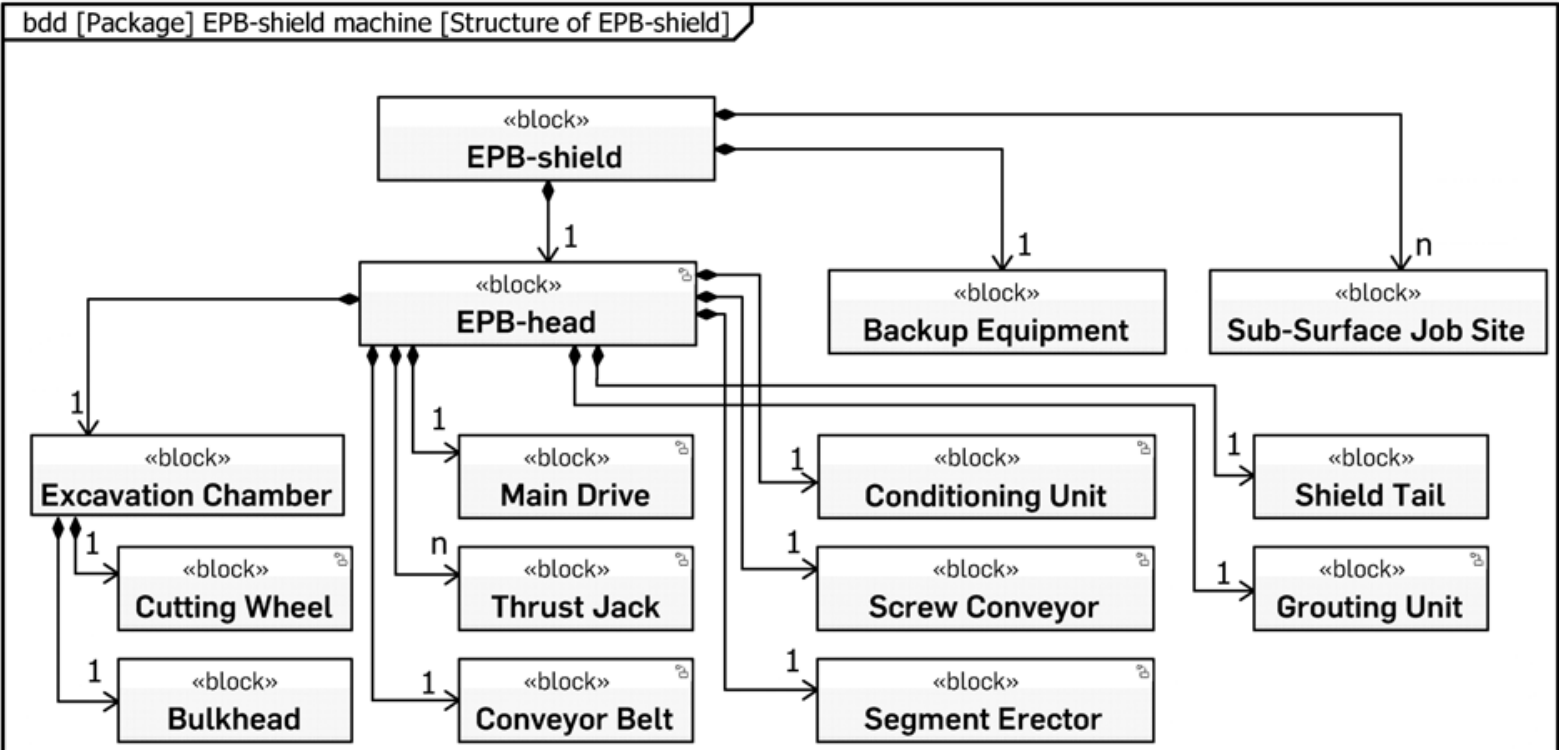

Figure 2: Block definition diagram of an EPB-shield

\subsection{Hybrid Simulation Approach}

The analysis of the interrelations between the TBM core processes and the allocated supply chain brought up a particular problem concerning the comparability: First, operational processes should be treated as actions performed at discrete time-intervals. In contrast to that, the material-flow of excavated soil exhibits the features of a continuous stream and is hardly abstractable to a specific moment or a single object. The delivery of segments for the ringbuild on the other hand is again dependent to a discrete event. For this reason, the developed simulation model combines two simulation techniques. The processes are modeled by application of the discrete-event paradigm. The propagation of the continuous flow of excavated soil is modeled with system dynamics.

Besides, the combination of the process-oriented state-machine and continuous system dynamics enables the possibility to simulate disturbances of the system without splitting the affected state beforehand. The system dynamic model of an element can be regarded as a "loading bar" that represents the progress of a specific task or condition.

In case a disturbance occurs, the state is left unchanged and the system dynamic model stores the current progress. The state can be re-entered after troubleshooting and the work is resumed at the former point of progress. This issue is illustrated in Figure 3. During the process execution of Excavating $100 \mathrm{~m}^{3}$ a disturbance occurs (after $60 \%$ ) and the state must be left unchanged. In conventional discrete-event simulation, the process is started all over again after fault remedy. Using the hybrid approach, the excavation is resumed at the former status. 
Rahm, Sadri, Koch, Thewes, and König

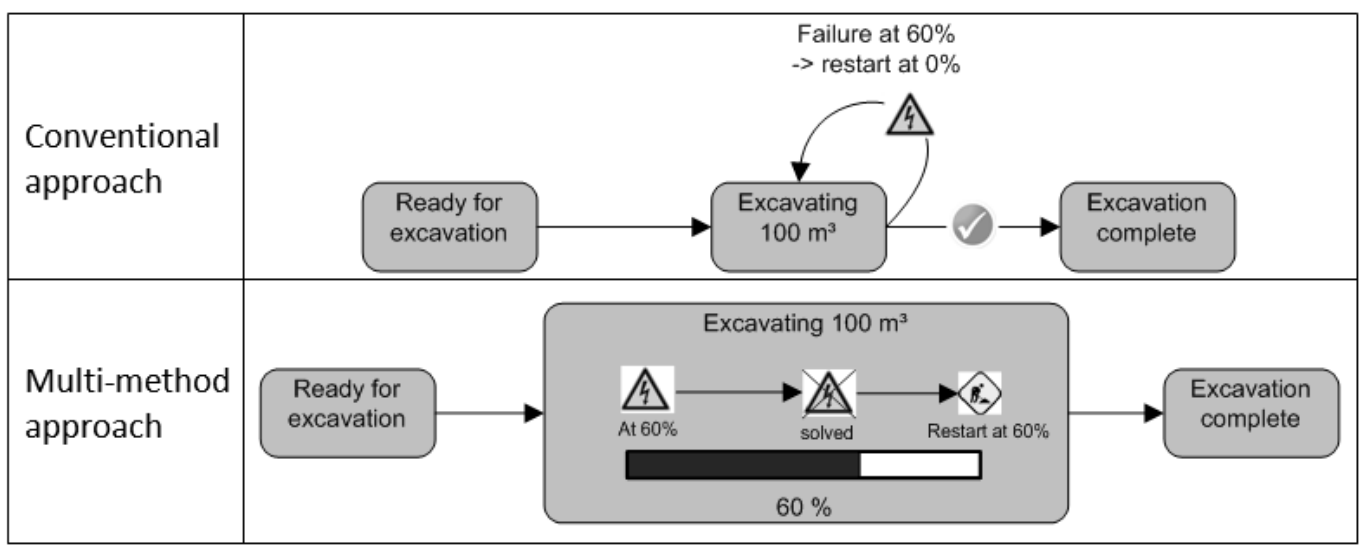

Figure 3: Comparison of conventional discrete-event simulation with the hybrid approach concerning disturbances while process execution.

\subsection{Implementation}

The multi-method simulation software AnyLogic by XJ Technologies (XJ Technologies 2012) provides the environment to implement the formalized model. The tool allows the simultaneous use of discreteevent, agent-based and system dynamic simulation.

The developed model is based on individual and adaptable simulation modules. This enables a flexible composition and easy parameterization of any envisaged project. The modules, represented by Active Object Classes (AOC) in AnyLogic, correspond to the pertinent blocks described with SysML. The possibility to consider alternating ground conditions is given. Following, detailed information about the implementation of the ambivalent simulation paradigms is presented.

In Figure 4 the internal composition of the AOC MainDrive is highlighted. The processes are modeled by state charts. The transition evOperate transfers the element from the state inactive into the state operating. In case the system works normally, the state operating is left via the transition evNormal. During a malfunction the auxiliary state outOfOrder is used to alternate the normal behavior. After fault remedy, the transition evRepaired is called and operating is re-entered.

The hybrid approach to simulate the material-flow, can also be used to handle the occurrences of disturbances. The internal "loading bar", provided by a system dynamic model, is used to measure the time span of undisturbed operation. The mean time between failures (MTBF) of the assembly group is calculated and assigned to the stock-variable nextMalfunction. This variable represents the remaining time until the next disturbance occurs. The flow-variable working reduces the stock by one, every time-unit the MainDrive is inside the state operating. Consequently the stock-variable totalWorkingTime is increased by one every time-unit of operation. Figure 4 displays a snapshot during a simulation run, where the MainDrive is momentarily in the state operating - visible by the thick border. Since the state operating is active, the flow-variable working is also active. The nextMalfunction will occur after further 5.166 working time-units of the MainDrive. The element was disturbed six times so far (numDisturbance $=6$ ). 
Rahm, Sadri, Koch, Thewes, and König

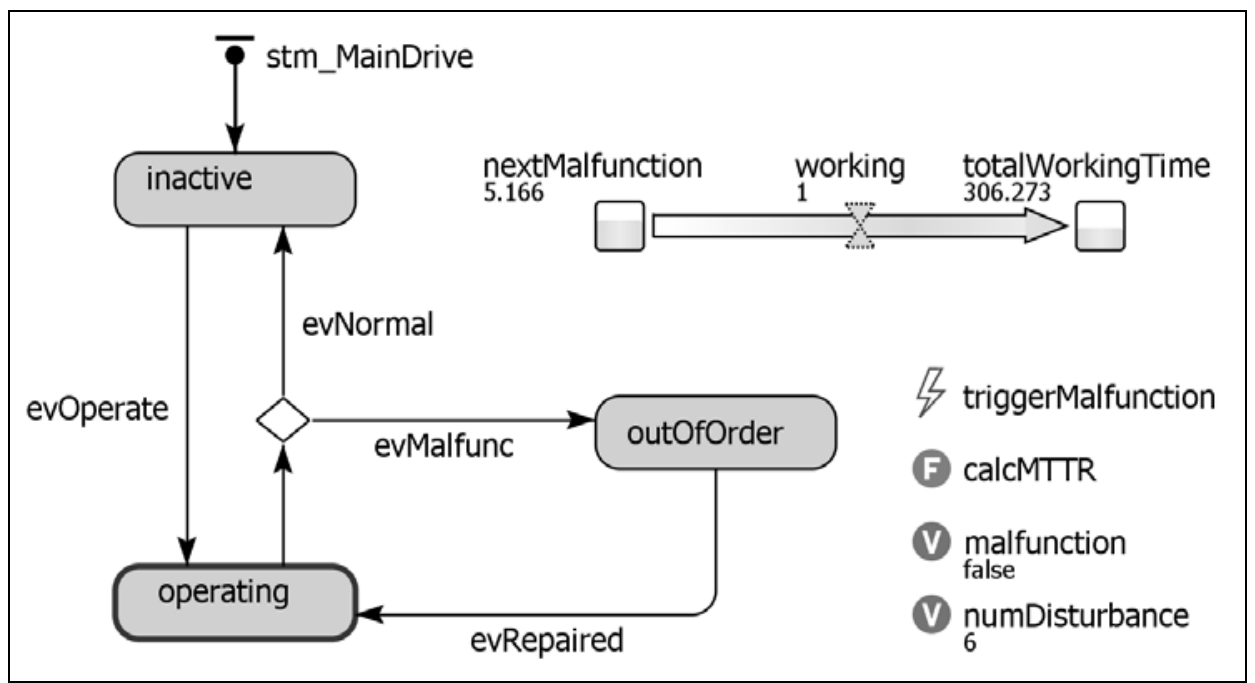

Figure 4: The AOC MainDrive during the state operating.

The disturbance occurs as soon as the value of nextMalfunction is zero. A trigger, observing this threshold value, transfers the system into the state outOfOrder. The mean time to recover (MTTR) is calculated by evaluating a normal-distribution. The result of this distribution is set as time-out to trigger the transition evRepaired. Another normal-distribution computes a new value for nextMalfunction and thus resets the "loading bar". Figure 5 depictures this issue. The system is currently in the state outOfOrder. The remaining duration of fault remedy is computed to be 2.81 time-units and a new value was assigned to the stock-variable nextMalfunction (i.e. 63.072). Consequently, the number of disturbances occurred, is updated to seven.

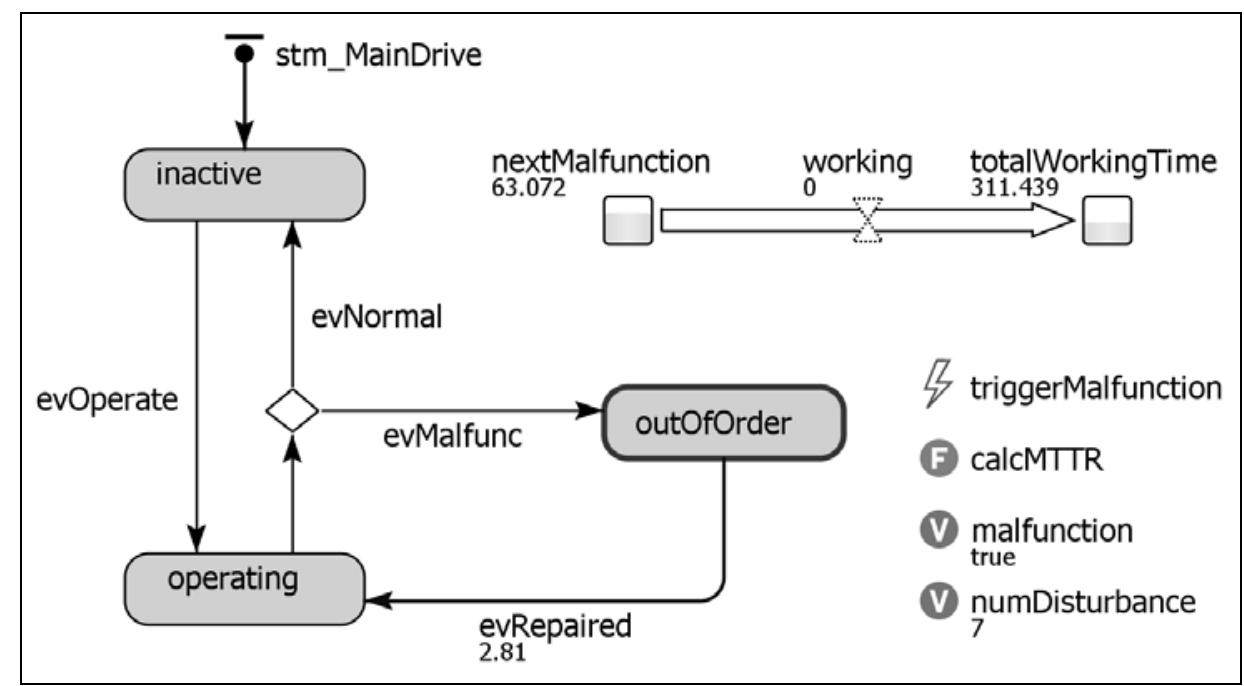

Figure 5: The $A O C$ MainDrive during a disturbance. 


\section{CASE STUDY}

A case study is presented in order to illustrate the developed approach. The case study comprises two simulation experiments. Disturbances are not considered within the first experiment. The impact of standstills on the project performance was analyzed by the second experiment. Currently, some simplifications and abstractions have been made. Thus, the supply chain is not considered yet. As a consequence, processes do not fail due to a shortage of required material. The muck handling or landfill is neither considered, so that not-dispatched material does not block any process. Furthermore, the geological conditions of a layer are defined to be constant, without any pockets of hard rocks and exactly as prognosticated in the preliminary planning. Additionally, the shut-down and initialization sequences of the TBM system are not modeled. And lastly, no maintenance or shift regulations are specified. The project is progressing twenty-four seven.

\subsection{Project Description}

The project simulated in this case study is based on a real tunneling project. Corresponding specifications and dimensions were extracted partially. The geology of the marked-out route enables the use of an EPBshield. The total length of the tunnel to be constructed underground is 8000 meters. Table 1 summarizes the main parameters of the project.

Table 1: Parameter setup of the simulation model

\begin{tabular}{lll}
\hline Parameter & Value & Unit \\
\hline Tunnel length & 8000 & $\mathrm{~m}$ \\
Diameter machine & 10 & $\mathrm{~m}$ \\
Width of segment & 2 & $\mathrm{~m}$ \\
Number of segments per ring & 7 & $\mathrm{pcs}$ \\
\hline
\end{tabular}

The advancement rate of the machine is determined according to current soil conditions. In Figure 6 the prognosticated soil profile is illustrated in simplified terms.

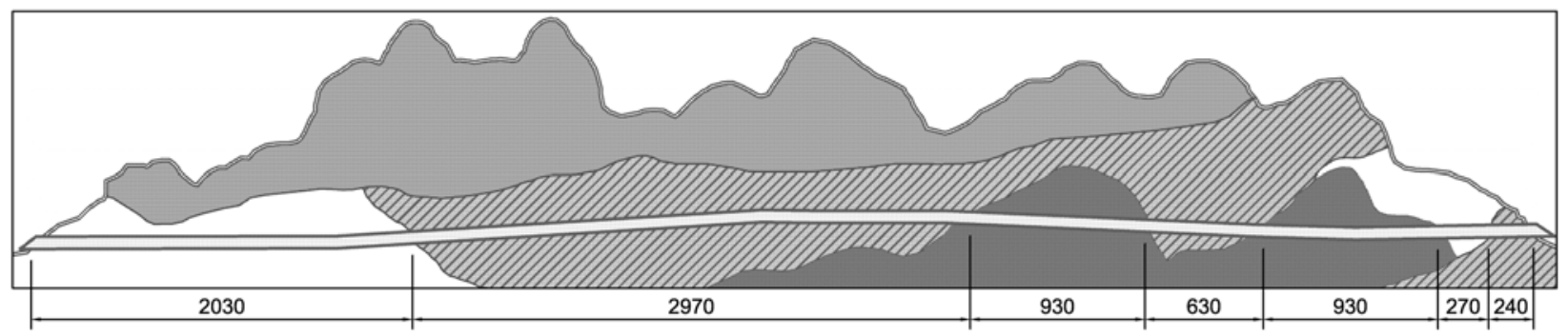

Figure 6: Geological formations along marked-out route of the case study project

Large sections of undisturbed soil layers are visible. Within these sections a specific range of advancement per time unit $(\mathrm{mm} / \mathrm{min})$ is assumed. This advancement rate is determined by a triangular distribution considering a minimum, maximum, and mean value. These simplified values were defined after analyzing the existing project data. The specific values of the layers are displayed in Table 2 . In order to dynamize the simulation, this distribution is evaluated for every segment instead of once at the beginning of a layer. 
Rahm, Sadri, Koch, Thewes, and König

Table 2: Input values for determination of the advancement rate

\begin{tabular}{lllll}
\hline Section & $\begin{array}{l}\text { Length } \\
{[\mathrm{m}]}\end{array}$ & $\begin{array}{l}\text { Minimum } \\
{[\mathrm{mm} / \mathrm{min}]}\end{array}$ & $\begin{array}{l}\text { Mean } \\
{[\mathrm{mm} / \mathrm{min}]}\end{array}$ & $\begin{array}{l}\text { Maximum } \\
{[\mathrm{mm} / \mathrm{min}]}\end{array}$ \\
\hline 1 & 2030 & 20 & 25 & 40 \\
2 & 2970 & 30 & 40 & 50 \\
3 & 930 & 10 & 15 & 25 \\
4 & 630 & 30 & 40 & 50 \\
5 & 930 & 10 & 15 & 25 \\
6 & 270 & 20 & 25 & 40 \\
7 & 240 & 30 & 40 & 50 \\
\hline
\end{tabular}

The ringbuild, as the second core process of mechanized tunneling in soft ground, has a crucial influence to the project performance. For this reason, this process was considered in the simulation model. The assembly of the ring is made up of seven single segments. Although the segments feature partially different shapes, the presented simulation model does not distinguish the particular segments yet. All segments are installed in the same way. The timescale of a single installation is determined again by a triangular distribution. Measurements of the duration of a ring assembly were analyzed to extract the input data for the distribution. The minimum, maximum and mean values (see Table 3) of the period for one segment installation correspond to an overall duration of around 45 minutes to assemble one complete ring.

Table 3: Duration of installation of one segment per ring

\begin{tabular}{lll}
\hline Parameter & Value & Unit \\
\hline Minimum duration & 6 & min \\
Mean duration & 7.5 & min \\
Maximum duration & 9 & min \\
\hline
\end{tabular}

The first simulation experiment was executed without any disturbances. The second experiment setup is chosen in such a way, that disturbances occur solely in the element Main Drive. A malfunction of this element inevitably leads to a standstill of the whole excavation process. Obviously, this constellation is still highly abstract compared to the real TBM system, where numerous elements can cause a delay in the predicted advancement rate. In prospective publications, the influence of multitudinous disturbances with varying extent will be the focus. Nevertheless, the implemented approach is applicable to adequately consider disturbances of TBM systems in a simulation model.

The MTBF is generated by normal distribution. The same is true for the mean time to recover. The restrictions of an underground TBM project entail the risk that the repair faces unforeseen complications and takes longer time than usual. This possibility is also considered within the simulation model. The specific input values of the distributions, condensed in Table 4, are assumptions made for this publication. 
Rahm, Sadri, Koch, Thewes, and König

Table 4: Configuration of disturbance simulation

\begin{tabular}{llll}
\hline Distribution for & Parameter & Value & Unit \\
\hline MTBF & $\mu$ & 50 & $\mathrm{~h}$ \\
& $\sigma$ & 10 & $\mathrm{~h}$ \\
\hline MTTR (probability 80\%) & $\mu$ & 5 & $\mathrm{~h}$ \\
& $\sigma$ & 1 & $\mathrm{~h}$ \\
\hline MTTR (probability 16\%) & $\mu$ & 10 & $\mathrm{~h}$ \\
& $\sigma$ & 2 & $\mathrm{~h}$ \\
\hline MTTR (probability 4\%) & $\mu$ & 50 & $\mathrm{~h}$ \\
& $\sigma$ & 10 & $\mathrm{~h}$ \\
\hline
\end{tabular}

In order to eliminate freak values evoked by randomness, a Monte Carlo experiment of one thousand iterations is conducted for each case. This number of iterations is assumed to be sufficient to gather stable and averaged results. The parameters of machine and project remain constant during this Monte Carlo experiment. Solely the seed-value is varied. This variation randomizes the probability distributions, used to calculate advancement rate, time span of undisturbed operation, and duration of fault remedy.

\subsection{Simulation Experiments}

Figure 7 shows the production rate (number of rings assembled per day) of one random simulation run of the first experiment. The different soil layers are clearly visible by the partially severe changes in assembled rings per day. The peaks within one layer appear due to the strict counting of rings finished within 24 hours. For the disturbance-free case, where all materials are available if needed and no maintenance is necessary, the execution of the envisaged project has the duration of averaged 330 days. The fastest project was finished after 328 days. The longest duration computed by the Monte Carlo experiment is 332 days.

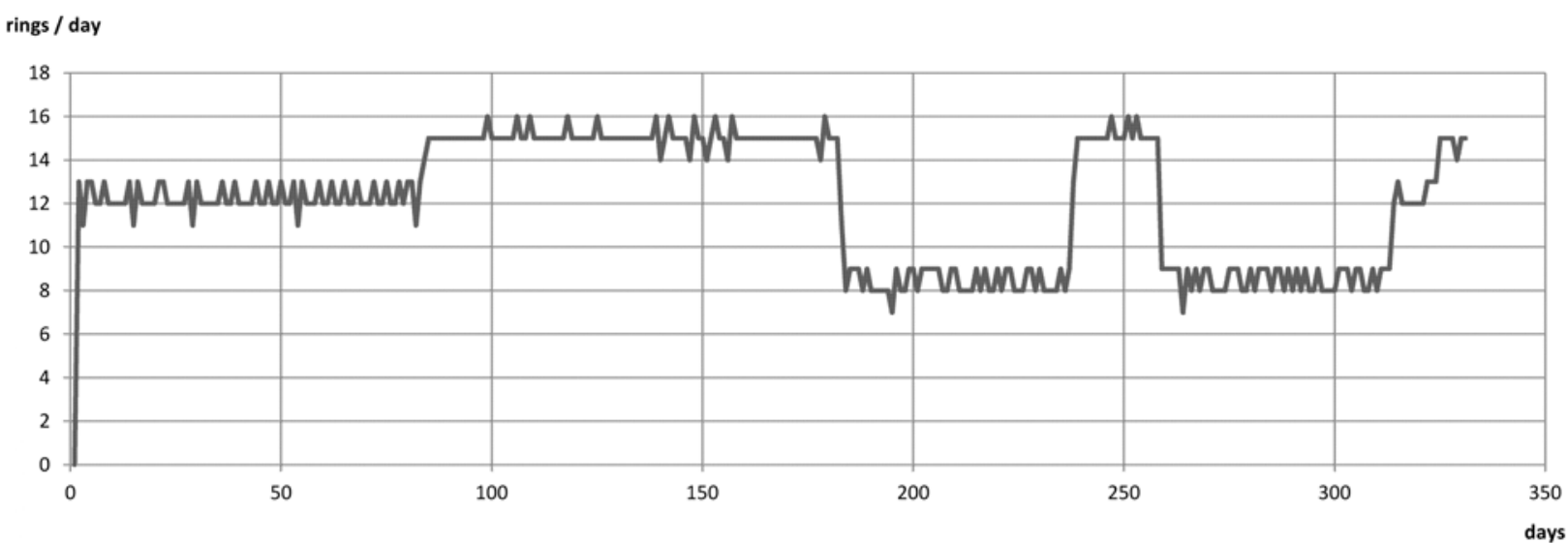

Figure 7: Rings per day without consideration of disturbances

Figure 8 displays the performance of the TBM system influenced by disturbances of the MainDrive. The production rate shows partially high incursions, including total standstills for longer than one whole day. 


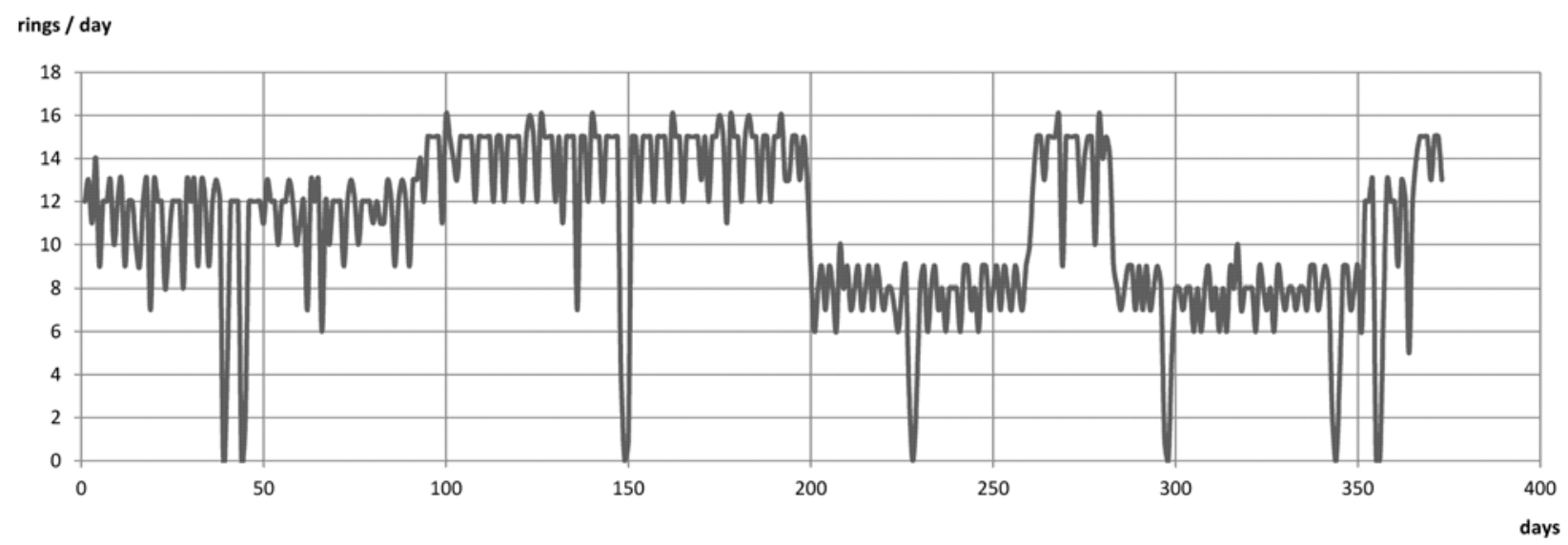

Figure 8: Rings per day considering disturbances

In this second case, the project duration is expectedly longer compared to the first case. After one thousand iterations, a mean period of 361 days attunes. Compared to the first case, this entails a prolongation of the project of about $9.5 \%$. The risk of non-scheduled costs resulting from such a prolongation is obvious. The minimum project duration of the one thousand iterations is 350 days. The longest duration computed is 378 days. This signifies a maximum project extension of 50 days, compared to the fastest undisturbed project execution.

\section{CONCLUSION}

This paper propounds the current status of an approach to predict the production rate of TBMs by application of two different simulation techniques. The combination of discrete and continuous simulation paradigms is described and the advantages are outlined. The implementation of a case study is provided to illustrate the method of operation.

Due to the early phase of the project, the simulation model is restricted to two core processes of tunneling. In future research the supply chain will be included. Furthermore, fuzzy constraints for underground construction works (e.g. soil conditions) will be integrated into the model. Furthermore, the impact of disturbances on critical elements and their effects to successors will be investigated in more detail. In addition to that, the shut-down and initialization sequences implicated by standstills will be included.

\section{ACKNOWLEDGMENTS}

Financial support was provided by the German Science Foundation (DFG) in the framework of project C3 of the Collaborative Research Center SFB 837. This support is gratefully acknowledged. 


\section{REFERENCES}

Alvarez Grima, M., A. Bruines, and P. N. Verhoef. 2000. "Modeling tunnel boring machine performance by neuro-fuzzy methods." In Tunnelling and Underground Space Technology 3:259-269.

Benardos, A. G., and D. C. Kaliampakos. 2004. "Modelling TBM perfomance with artificial neural networks." In Tunnelling and Underground Space Technology 6:597-605.

Budach, C., and M. Thewes. 2010. "Soil conditioning with foam during EPB Tunnelling." In Geomechanics and Tunnelling 3:256-267.

Chung, T. H., Y. Mohamed, and S. M. AbouRizk. 2006. "Bayesian updating application into simulation in the North Edmonton Sanitary Trunk tunnel project." In Journal of Construction Engineering and Management 132:882-894.

Donghai, L., Z. Yunqing, and K. Jiao. 2010. "TBM construction process simulation and performance optimization." In Transactions of Tianjin University 16:194-202.

Herrenknecht AG. 2008. "Herrenknecht - EPB Shield". Accessed Mai 21. http://www.herrenknecht.com/process-technology/machine-technology/epb-shield.html.

Herrenknecht, M., M. Thewes, and C. Budach. 2011. "The development of earth pressure shields: from the beginning to the present." In Geomechanics and Tunnelling 1:11-35.

König, M. 2011. "Robust construction scheduling using discrete-event simulation." In Proceedings of the 2011 ASCE International Workshop on Computing in Civil Engineering. Edited by Y. Zhu, R. Issa. 55-63. Reston, Virginia: American Society of Civil Engineering.

Leitner, W., and E. Schneider. 2005. "Operational modelling of advance rates for tunnel boring machines." In Felsbau 6:9-14.

Likhitruangsilp, V., and P. G. Ioannou. 2003. "Stochastic evaluation of tunnelling performance using discrete-event simulation." In Proceedings of the 2003 Construction Research Congress, Edited by K. R. Molenaar, and P. S. Chinowsky, 1-8. Reston, Virginia: American Society of Civil Engineers.

Maidl, B., M. Herrenknecht, U. Maidl, and G. Wehrmeyer. 2012. Mechanised shield tunnelling. Berlin: Ernst W. \& Sohn Verlag.

Object Management Group. 2010. "OMG Systems Modeling Language (OMG SysMLTM).” Accessed Mai 21. http://www.omg.org/spec/SysML/1.2/.

Ourdev, I., S. M. AbouRizk, and H. T. Al-Battaineh. 2007. "Simulation and uncertainty modelling of project schedules estimates." In Proceedings of the 2007 Winter Simulation Conference. Edited by J. Taw, R. Barton, S. Henderson, B. Biller, M. Hsieh, and J. Shortle, 2128-2133. Piscataway, New Jersey: Institute of Electrical and Electronics Engineers, Inc.

Ruwanpura, J. Y.,S. M. AbouRizk, and S. Fernando. 2001. "Special purpose simulation templates for tunnel construction operations." In Canadian Journal of Civil Engineering 28:222-237.

Thewes, M. 2007. "TBM Tunnelling Challenges - Redefining the State-of-the-Art - Keynote lecture at the 2007 World Tunnel Congress in Prague." In Tunnel 16:13-21.

Thewes, M. 2010. "Shield tunnelling technology for increasingly difficult site conditions." In Proceedings of the $11^{\text {th }}$ International Conference on Underground Construction. Edited by I. Hrdina, A. Butovič, and M. Hilar, 371-379. Prague: Czech ITA-AITES Tunnelling Association.

XJ Technologies. 2012. "Why AnyLogic simulation software?". Accessed Mai 21. http://www.xjtek.com/anylogic/why_anylogic/. 


\section{AUTHOR BIOGRAPHIES}

TOBIAS RAHM received diploma in management for construction, real estate and infrastructure at the Bauhaus-University in Weimar. Since 2011 he is working as research assistant at the Chair of Computing in Engineering at the Ruhr-University in Bochum, Germany. His research interests are in application of operational simulation techniques in the field of mechanized tunneling. He is currently intensifying his research within the Collaborative Research Center 837 announced by the German Research Foundation. His email address is tobias.rahm@ruhr-uni-bochum.de.

KAMBIZ SADRI is research assistant at the Institute for Tunneling and Construction Management at the Ruhr-University Bochum, Germany. He finished his master courses at the Ruhr-University Bochum, Germany. His research interests are model- and monitoring based optimization and quality control in mechanized tunneling using process simulation. He is currently intensifying his research within the Collaborative Research Center 837 announced by the German Research Foundation. His email address is Kambiz.Sadri@rub.de.

CHRISTIAN KOCH is a postdoctoral group leader at the chair/institute of Computing in Engineering at the Ruhr-Universität Bochum. In 2008, he received his doctorate in Civil Engineering from the BauhausUniversität Weimar for his work on distributed building information modeling. Since 2010, he leads the Computer Vision and Mixed Reality group that conducts research on vision-based IT support in several fields of construction, such as progress monitoring, condition assessment and facility maintenance. His email address iskoch@inf.bi.rub.de.

MARKUS THEWES is professor of Tunneling and Construction Management at the Ruhr-University Bochum. Before joining Ruhr-University Bochum in Germany, he was a design engineer for tunnel constructions, a geotechnical engineer for a TBM manufacturer, a jobsite manager for large tunneling projects, and a senior researcher at a private tunnel research foundation. The research work at his institute focuses on Technologies for Tunnel Boring Machines, Sprayed Concrete Applications, Process Simulation and Risk Management in Tunneling, Operation and Maintenance of Tunnels and Utility Lines, as well as Safety and Security issues of Underground Structures. Markus Thewes has 80 publications on tunneling issues. He is Vice President of the International Tunneling and Underground Space Association. His emailistlb@rub.de.

MARKUS KÖNIG is professor of Computing in Engineering at Ruhr-University Bochum, Germany. Previously, he was Assistant Professor of Theoretical Methods for Project Management at BauhausUniversity Weimar, Germany. He obtained his Ph.D. in Civil Engineering from Leibniz-University Hanover, Germany in 2003. His research interests include construction simulation and optimization, product and process management, knowledge management in construction, intelligent computing in engineering and computational steering. His email is koenig@inf.bi.rub.de. 